\title{
Article \\ Duration of Reduced CA19-9 Levels Is a Better Prognostic Factor Than Its Rate of Reduction for Unresectable Locally Advanced Pancreatic Cancer
}

\author{
Ko Tomishima (1), Shigeto Ishii (1), Toshio Fujisawa, Muneo Ikemura, Hiroto Ota, Daishi Kabemura, Mako Ushio,

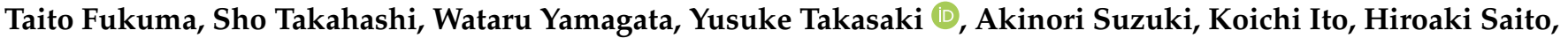 \\ Akihito Nagahara and Hiroyuki Isayama * (D)
}

Citation: Tomishima, K.; Ishii, S.; Fujisawa, T.; Ikemura, M.; Ota, H.; Kabemura, D.; Ushio, M.; Fukuma, T.; Takahashi, S.; Yamagata, W.; et al. Duration of Reduced CA19-9 Levels Is a Better Prognostic Factor Than Its Rate of Reduction for Unresectable Locally Advanced Pancreatic Cancer. Cancers 2021, 13, 4224. https:// doi.org/10.3390/cancers13164224

Academic Editor: Masakatsu Tsurusaki

Received: 23 July 2021

Accepted: 20 August 2021

Published: 22 August 2021

Publisher's Note: MDPI stays neutral with regard to jurisdictional claims in published maps and institutional affiliations.

Copyright: (c) 2021 by the authors. Licensee MDPI, Basel, Switzerland. This article is an open access article distributed under the terms and conditions of the Creative Commons Attribution (CC BY) license (https:// creativecommons.org/licenses/by/ $4.0 /)$.
Department of Gastroenterology, Graduate School of Medicine, Juntendo University, 3-1-3 Hongo, Bunkyo-ku, Tokyo 113-0033, Japan; tomishim@juntendo.ac.jp (K.T.); sishii@juntendo.ac.jp (S.I.); t-fujisawa@juntendo.ac.jp (T.F.); m-ikemura@juntendo.ac.jp (M.I.); h-ota@juntendo.ac.jp (H.O.); d.kabemura.nc@juntendo.ac.jp (D.K.); m-ushio@juntendo.ac.jp (M.U.); t.fukuma.vh@juntendo.ac.jp (T.F.); sho-takahashi@juntendo.ac.jp (S.T.); w.yamagata.mx@juntendo.ac.jp (W.Y.); ytakasa@juntendo.ac.jp (Y.T.); suzukia@juntendo.ac.jp (A.S.); kitoh@juntendo.ac.jp (K.I.); hiloaki@juntendo.ac.jp (H.S.); nagahara@juntendo.ac.jp (A.N.)

* Correspondence: h-isayama@juntendo.ac.jp

Simple Summary: Upon diagnosis, about 35\% of patients have initially unresectable locally advanced pancreatic cancer. The prognosis of these patients is still poor. Chemotherapy alone has been generally accepted as a standard therapeutic approach. However, clinical decision-making processes have not been established for aggressive treatments such as surgery and chemoradiotherapy in patients with a response and stable case of initially unresectable locally advanced pancreatic cancer. In the current study, we evaluated the reduction rate and duration of carbohydrate antigen (CA) 19-9 within 6 months as long-term survival. Cases of over 44\% CA 19-9 reduction only one month from the baseline after treatment were not significantly associated with overall survival. On the other hand, more than 3 months of over 44\% CA 19-9 reduction was significantly associated with prognosis, which is the same as the occurrence of distant metastasis. Multidisciplinary treatment focus on local treatment is expected in these selected patients.

Abstract: A decrease in carbohydrate antigen (CA) 19-9 levels has been proposed as a prognostic marker for survival and recurrence in patients with pancreatic cancer. We evaluated the association between duration of reduced CA 19-9 levels during 6 months after treatment and long-term survival for 79 patients with unresectable locally advanced pancreatic cancer (LAPC). We calculated the differences between pretreatment and monthly CA19-9 levels. We categorized 71 patients with decreases in CA19-9 levels into three groups based on the duration of these reduced levels $(>2,>3$, and $>4$ months). The cut-off level for long-term (more than 2 years) survival was identified as a $44 \%$ reduction from the baseline, using a ROC curve. A reduction duration $>2$ months was not associated with overall survival $(p=0.1)$, while $>3$ months was significantly associated with survival $(p=.04)$. In multivariate analysis, a reduction duration $>3$ months predicted a good long-term prognosis (odds ratio $=5.75 ; 95 \%$ confidence interval $=1.47-22.36 ; p<0.01$ ). In patients with unresectable LAPC, the duration of reduced CA19-9 levels for more than 3 months, rather than the rate of reduction in CA19-9 levels, during 6 months after treatment was significantly associated with good prognosis.

Keywords: CA 19-9; pancreatic cancer; prognostic factor

\section{Introduction}

Pancreatic cancer has an increasing incidence and the highest mortality out of all gastrointestinal cancers [1]. Complete surgical resection of pancreatic cancer is associated with the best outcomes. Unfortunately, less than $20 \%$ of patients are surgical candidates, and 
most have unresectable cancer [2]. Recent studies have demonstrated superior outcomes for surgery after neoadjuvant chemotherapy (NACT) compared to upfront surgery for patients with borderline resectable pancreatic cancer (BRPC) or locally advanced pancreatic cancer (LAPC) [3-5]. The optimal timing for surgery is 240 days after beginning NACT. Preoperative chemotherapy (CTx) or chemoradiotherapy (CRTx) prolongs survival in pancreatic cancer patients $[6,7]$. However, $20-70 \%$ of patients are surgical candidates after CTx or CRTx, and indications for surgery vary between institutions [8-10].

More than $80 \%$ of patients with advanced pancreatic cancer have increased levels of the serum tumor marker carbohydrate antigen (CA) 19-9 [11]. The levels of CA 19-9 reflect the dynamic effects of CTx or CRTx without patients negative for the Lewis blood group phenotype. CA 19-9 levels after NACT and surgical resection correlate with the R0 resection rate, histopathological response, and survival rates [12-15]. CA 19-9 levels also correlate with the survival of patients with advanced pancreatic cancer [16-19]. However, previous studies did not exclude patients with metastasis and used different CA 19-9 cut-off levels. Therefore, the optimal cut-off values for CA 19-9 levels are unclear. The cut-off values for CA 19-9 may be used to select LAPC patients with a good chance of survival for further treatment. In addition, prognostic factors for LAPC patients should be determined.

In this retrospective study, we determined the changes in CA 19-9 levels of unresectable LAPC patients during 6 months after CTx or CRTx.

\section{Materials and Methods}

\subsection{Serum Levels of CA 19-9}

CA 19-9 levels were measured monthly for 6 months after beginning treatment (upper limit: $37 \mathrm{U} / \mathrm{mL}$ ). Patients negative for the Lewis blood group phenotype, defined as undetectable CA 19-9 throughout the study, were excluded [20]. In cases of obstructive jaundice, biliary drainage was performed prior to obtaining a serum sample. Changes in CA 19-9 levels from baseline were assessed using the reduction ratio, calculated as: currentCA 19-9 level/pretreatment CA 19-9 level (RR-C; reduction ratio of CA 19-9). For example, RR-C 3 months after the treatment was defined as: 3-month CA 19-9 level/pretreatment CA 19-9 level. Within normal limit in CA 19-9 levels after treatment was observed in 11 patients, who were included in the CA 19-9 decrease group.

\subsection{Patients}

We included 134 patients who were histologically diagnosed with unresectable LAPC, including initially BRCP, and received CTx or CRTx at Juntendo University Hospital between December 2005 and June 2020. We included initially BRPC cases that were progressed in spite of initial treatment. We excluded patients who received best supportive care (BSC) due to performance status of 3 or $4(n=5)$, being followed up for less than 3 months $(n=28)$, undergoing conversion surgery $(n=15)$, or being negative for the Lewis blood group phenotype $(n=7)$. After exclusion of these patients, 79 were included as finally unresectable locally advanced pancreatic cancer in the study (Figure 1). All cases of pathological phenotype were adenocarcinoma except one case of adenosquamous carcinoma. In addition, the relationships between CA 19-9 levels and long-term survival (defined as survival for $>2$ years) were also evaluated. Patients with a decrease in CA 19-9 levels $(n=71)$ were categorized into three groups based on the duration of CA19-9 reduction (i.e., 2, 3, or 4 months) (Figure 1). The medical records of patients were reviewed to obtain the age, sex, performance status, tumor location, CA19-9 levels (initial level, reduction rate, and reduction duration), tumor size, initial resectability, and treatment. The study protocol was approved by the Institutional Review Board of Juntendo University Hospital (IRB No: 20-007). 




Figure 1. Patients flow chart. LAPC: locally advanced pancreatic cancer; BSC: best supportive care; RR-C: reduction ratio of CA 19-9.

\subsection{Resectability and Treatment}

BRPC was defined as a tumor that was in contact with the common hepatic artery (CHA), without extension into the celiac axis (CA) or hepatic artery bifurcation; in contact with $\leq 180^{\circ}$ of the circumference of the superior mesenteric artery (SMA); in contact with $>180^{\circ}$ of the circumference of the superior mesenteric vein or portal vein (PV); or in contact with $\leq 180^{\circ}$ of the circumference of either the superior mesenteric or portal vein, with an irregular contour or venous thrombosis but possibility of reconstruction [21]. LAPC was defined as a tumor that had $>180^{\circ}$ contact with or invasion of SMA or PV and extended beyond the lower border of duodenum; $>180^{\circ}$ contact with or invasion of SMA or CA; or contact with or invasion of $\mathrm{CHA}$, proper hepatic artery, $\mathrm{CA}$, or aorta. These patients were not offered surgery because vascular invasion does not improve in the course of treatment.

The CTx or CRTx regimens were decided by the attending physician. The patients were evaluated using CA 19-9 levels and serial abdominal computed tomography (CT), magnetic resonance, and positron emission tomography (PET) imaging. CT images were evaluated using the modified Response Evaluation Criteria in Solid Tumors [22].

\subsection{Statistical Analysis}

The Kaplan-Meier method was used to estimate the overall survival (OS). OS was calculated based on the dates of diagnosis and death. Statistical differences in OS were analyzed using the two-tailed log-rank test. Fisher's exact test was used to compare qualitative data, where appropriate. Logistic regression was used for multivariate analysis, after adjusting for all potential confounding factors. Differences were assumed to be significant at $p<0.05$.

\section{Results}

\subsection{Patient Characteristics}

Table 1 summarizes the baseline patient characteristics. The median age of the study participants was 68 (36-87) years. There were 32 females (41\%) and 47 males (59\%). Most participants had a performance status of $0(n=63,80 \%)$, while others had 1 or 2 $(n=16,20 \%)$. The median pretreatment CA $19-9$ was $182(10-21,084) \mathrm{U} / \mathrm{mL}$. There was no significant difference between initial CA $19-9$ and overall survival $(p=0.45)$. The tumors were located in the pancreatic head $(n=41,52 \%)$ or tail $(n=38,48 \%)$, and the median tumor size was $32(12-64) \mathrm{mm}$. The tumors were BRPC $(n=22,28 \%)$ or LAPC $(n=57,72 \%)$, with invasion into the CA $(n=33,44 \%)$, SMA $(n=36,45 \%)$, CHA $(n=3,4 \%)$, or PV $(n=5,7 \%)$. As for BRPC, 19 cases, in contact with $\leq 180^{\circ}$ of artery, received chemotherapy, and three 
cases, in contact with $>180^{\circ}$ of portal vein, also received chemotherapy because of tumor diameter (more than $40 \mathrm{~mm}$ ). CTx was administered to 63 patients $(80 \%)$, and CRTx was given to 16 patients $(20 \%)$. The CTx regimen consisted of gemcitabine plus nab-paclitaxel $(n=41)$, gemcitabine $(n=13)$, gemcitabine plus S-1 $(n=4, \mathrm{GS}), \mathrm{S}-1(n=3)$, or FOLFIRINOX $(n=2)$. CRTx patients received GS plus radiotherapy (GS-RT), with daily fractions of 1.8 Gy (total dose: 50.4 Gy over 5.5 weeks). Gemcitabine $\left(200 \mathrm{mg} / \mathrm{m}^{2}\right)$ was administered weekly for 6 weeks. S-1 ( $80 \mathrm{mg} / \mathrm{m}^{2} /$ day) was administered orally twice a day on days 1-14 and 22-35. Progression-free survival and OS were 8 (3-74) and 17 (4-128) months, respectively.

Table 1. Patient characteristics $(n=79)$.

\begin{tabular}{|c|c|c|}
\hline Factors & $n(\%)$ & Median (Range) \\
\hline Age, years & & $68(36-87)$ \\
\hline Sex, female/male & $32(41) / 47(59)$ & \\
\hline PS, $0 / 1,2$ & $63(80) / 16(20)$ & \\
\hline Pre-CA19-9, U/mL, & & $182(10-21084)$ \\
\hline Location, Head/Body-tail & $41(52) / 38(48)$ & \\
\hline Tumor size, $\mathrm{mm}$ & & $32(12-64)$ \\
\hline Resectability, BR/LA & $22(28) / 57(72)$ & \\
\hline Invasion, (CA/SMA/CHA/PV) & $35(44) / 36(45) / 3(4) / 5(7)$ & \\
\hline 1st Treatment, CTx/CRTx & $63(80) / 16(20)$ & \\
\hline CTx regimen & $\begin{array}{c}\text { GnP } 41, \text { Gem 13, GS 4, } \\
\text { S1 3, FFX } 2\end{array}$ & \\
\hline CRTx regimen & $\mathrm{Gem}+\mathrm{S} 1+\mathrm{RT}$ & \\
\hline PFS, month & & $8(3-74)$ \\
\hline OS, month & & $17(4-128)$ \\
\hline
\end{tabular}

PS: performance status; BR: borderline resectable, locally advanced; CA: celiac axis; SMA: superior mesenteric artery; CHA: common hepatic artery; PV: portal vein; CTx: chemotherapy; CRTx: chemoradiotherapy; PFS: progression free survival; OS: overall survival; GnP: gemcitabine plus nab-paclitaxel; Gem: gemcitabine; GS: gemcitabine plus S-1; FFX: FOLFIRINOX; RT: radiotherapy.

\subsection{CA19-9 Reduction Rate and Survival}

CA19-9 decreased from the baseline for $71(90 \%)$ patients on at least one occasion over the 6 months after treatment $(\mathrm{RR}-\mathrm{C}<0)$. Conversely, CA19-9 increased for eight $(10 \%)$ patients (RR-C $\geq 1)$. RR-C $<1$ was significantly associated with a better OS compared to RR-C $\geq 1$ (median OS $21 \mathrm{~m}$ vs. $12 \mathrm{~m}, p=0.02$, respectively). The CA19-9 cut-off value for long-term ( $>24$ months) prognosis and RR-C were defined on the basis of $44 \%$ reduction from the ROC curve $(75 \%$ of true positive fraction, $37 \%$ of false positive fraction, odds ratio 5.1). During 6 months after treatment, CA19-9 levels decreased for 71 patients, but improved OS was not observed in 64 (90\%) of these patients who had RR-C $>44 \%$ (median OS $21 \mathrm{~m}$ vs. $9 \mathrm{~m}, p=0.06$ ) (Figure 2).

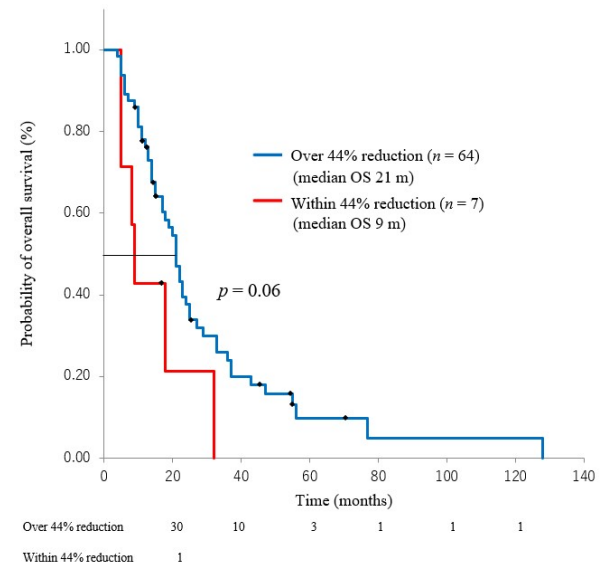

Figure 2. Among CA 19-9 reduction, relationship between within $44 \%$ reduction and over $44 \%$ reduction. 


\subsection{CA19-9 Reduction Duration and Overall Survival or Metastasis}

Patients were categorized into three groups based on the duration of CA19-9 reduction (>2 months: $n=57,80 \%$; $>3$ months: $n=48,68 \%$; >4 months: $n=46,65 \%$ ) (Figure 1). We evaluated the relationships between these durations and OS. Reduction duration $>2$ months was not associated with OS, while $>3$ and $>4$ months were associated with OS (22 and 15 months, $p=0.04 ; 23$ and 14 months, $p=0.01$, respectively) (Figure 3). As for time to distant metastasis, we compared $>3$ months reduction and other cases. RR-C $<0.56$ lasting for $>3$ months during the first 6 months after treatment predicted distant metastases $(p=0.01)$ (Figure 4).
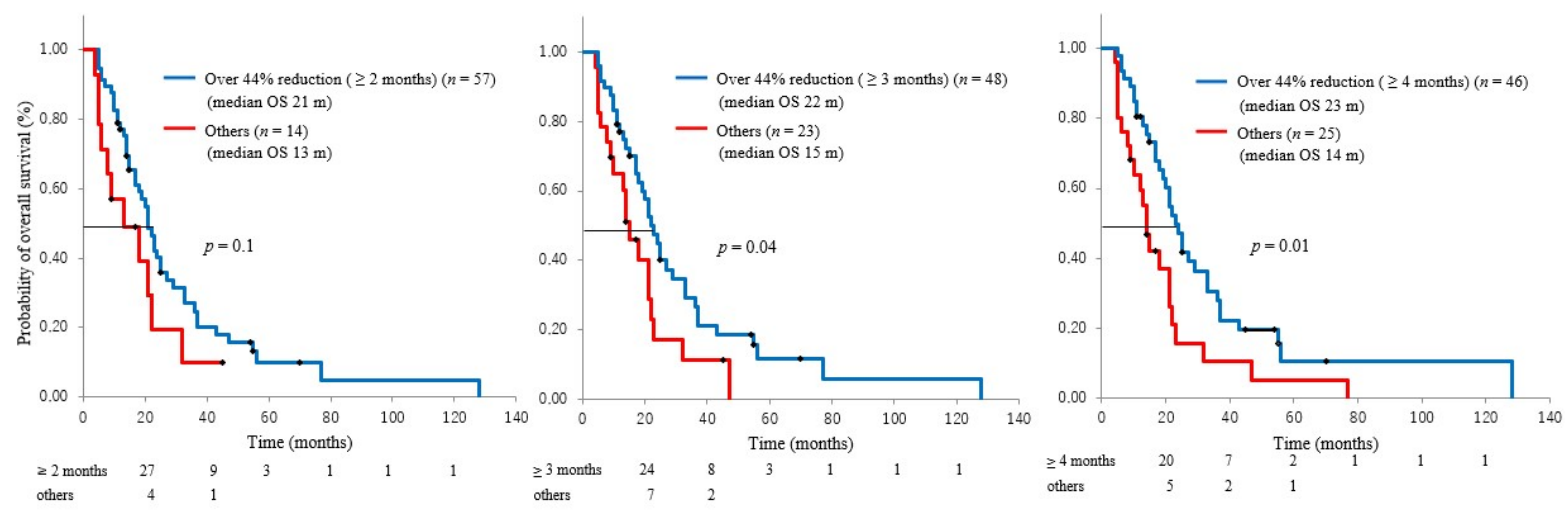

Figure 3. Among CA 19-9 reduction, relationship between $>44 \%$ reduction of these durations ( $>2$ months, $>3$ months, and $>4$ months) and OS.

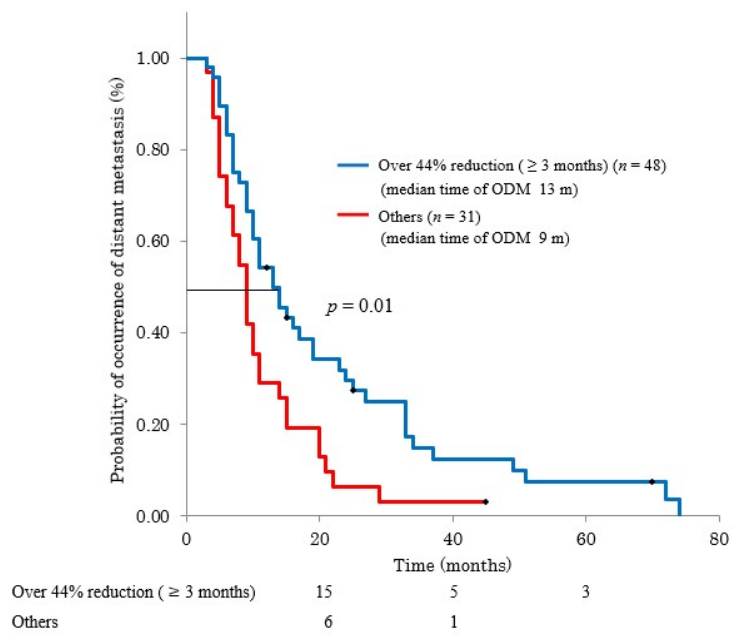

Figure 4. Relationship between $>44 \%$ reduction duration ( $>3$ months) and ODM. (ODM: occurrence of distant metastasis).

\subsection{Factors Related to Long-Term Survival}

Patients were divided into long-term ( $n=22$, LS group) and other survivors ( $n=57$, not LS group). Univariate analysis was performed for the effects of background factors and treatment methods on OS. Univariate analysis revealed that CRTx and CA 19-9 reduction $>44 \%$ for $>3$ months was significantly associated with long-term OS $(p=0.03, p<0.01)$ (Table 2). Multivariate analysis revealed that CA 19-9 reduction $>44 \%$ for $>3$ months independently predicted OS (odds ratio $5.75,95 \%$ CI 1.48-22.4, $p=0.01$ ). CRTx was also associated with increased long-term OS, albeit without statistical significance (odds ratio $2.63,95 \%$ CI 0.83-8.26, $p=0.09$ ) (Table 2). 
Table 2. Univariate and multivariate analysis for long term survival more than 2 years.

\begin{tabular}{|c|c|c|c|c|c|c|}
\hline \multirow[t]{2}{*}{ Variable } & \multicolumn{2}{|c|}{$\begin{array}{l}\text { Univariate } \\
\text { Analysis }\end{array}$} & \multicolumn{4}{|c|}{$\begin{array}{c}\text { Multivariate } \\
\text { Analysis }\end{array}$} \\
\hline & $\begin{array}{c}\text { LS } \\
(n=22)\end{array}$ & $\begin{array}{l}\text { not LS } \\
(n=57)\end{array}$ & $\begin{array}{c}p \\
\text { Value }\end{array}$ & $\begin{array}{l}\text { Odds } \\
\text { Ratio }\end{array}$ & $95 \%$ CI & $\begin{array}{c}p \\
\text { Value }\end{array}$ \\
\hline $\begin{array}{l}\text { Age (year) } \\
\geq 65 /<65\end{array}$ & $14 / 8$ & $38 / 19$ & 0.80 & & & \\
\hline Gender $\quad$ Male/Female & $15 / 7$ & $32 / 25$ & 0.30 & & & \\
\hline $\begin{array}{l}\text { Performance status } \\
\qquad \geq 1 / 0\end{array}$ & $2 / 20$ & $16 / 41$ & 0.07 & 0.35 & $0.07-1.81$ & 0.21 \\
\hline Location Head/Body-Tail & $12 / 10$ & $29 / 28$ & 0.80 & & & \\
\hline $\begin{array}{r}\text { Tumor Size }(\mathrm{cm}) \\
\qquad \geq 3 /<3\end{array}$ & $14 / 8$ & $35 / 22$ & 0.80 & & & \\
\hline $\begin{array}{r}\text { Initial Resectability } \\
\text { BR/LA }\end{array}$ & $6 / 16$ & $16 / 41$ & 0.9 & & & \\
\hline $\begin{array}{l}\text { Treatment } \\
\qquad \text { CRTx/CTx }\end{array}$ & $10 / 12$ & $12 / 45$ & 0.03 & 2.63 & $0.83-8.26$ & 0.09 \\
\hline CA19-9 & & & & & & \\
\hline $\begin{array}{l}\text { Initial level } \\
\qquad \geq 37 /<37\end{array}$ & $18 / 4$ & $45 / 12$ & 1.00 & & & \\
\hline $\begin{array}{l}\text { Reduction cases } \\
\qquad \geq 1 /<1\end{array}$ & $0 / 22$ & $8 / 49$ & 0.09 & & & \\
\hline Duration of $44 \%$ reduction & & & $<0.01$ & 5.75 & $\begin{array}{l}1.47- \\
22.36\end{array}$ & 0.01 \\
\hline$\geq 3 \mathrm{~m} /$ Others & $19 / 3$ & $29 / 28$ & & & & \\
\hline
\end{tabular}

\section{Discussion}

Treatment of pancreatic cancer with distant metastases has improved with the use of chemotherapy. CA 19-9 is suitable for the dynamic assessment of effects of CTx or CRTx. Several studies have reported that CA 19-9 levels correlate with OS in patients with advanced pancreatic cancer. Hess et al. reported that pretreatment serum levels of CA 19-9 independently predict OS. However, in a large multicenter cohort study, a decrease in CA 19-9 levels 2 months after chemotherapy was not associated with OS [18]. The cohort study differed from our study in terms of chemotherapy regimen (gemcitabine and capecitabine in that study) and disease stage. Other studies have reported a positive association between improved OS with an early decrease in CA 19-9 levels in unresectable pancreatic cancer patients [16,17]. Treatment effects observed 8 weeks after the treatment predicted tumor control at 12 weeks and future treatment effects. The time interval from start of treatment to lowest CA 19-9 levels was longer in patients without metastases compared to those with metastases [19]. Previous studies included patients with advanced pancreatic cancer, with or without metastasis, but did not report long-term survival. CA 19-9 has recently been reported to be a predictor for better outcomes after NACT. In patients with LAPC who received NACT, pretreatment CA 19-9 levels did not affect OS, but post-treatment normalization of CA 19-9 predicted good outcomes [23,24]. Similarly, a sustained decrease in CA 19-9 levels predicted better outcomes in BRPC patients who received NACT [25].

The optimal treatment strategy for unresectable LAPC is controversial. We retrospectively studied 79 patients with unresectable LAPC, including initially BRCP, who were followed up for more than 3 months. BRPC is neither clearly resectable nor clearly unresectable but rather implies a greater chance of incomplete resection in the setting of upfront surgery. Additionally, many groups have proposed definitions; however, there is not yet a universally accepted definition of BRPC [26]. We included initially BRPC cases that were progressed in spite of initial treatment and categorized "unresectable cases after initial treatment" as "unresectable locally advanced pancreatic cancer" in this study. 
Progression-free survival and OS were 8 and 17 months, respectively, and similar to those in patients treated with gemcitabine plus S-1 therapy for LAPC (11.76 m vs. $16.41 \mathrm{~m})$ [27]. CA 19-9 levels are affected by cholangitis or cholestasis. Therefore, we measured the CA 19-9 levels after treating these, after serum levels of bilirubin were within the normal range $(<2.0 \mathrm{mg} / \mathrm{dL})$.

Analysis of the RR-C changes in patients who survived $<12,12-24$, or $>24$ months demonstrated that CA 19-9 levels decreased the most in the first 6 months (Figure 5). In patients who survived $<12$ months, CA 19-9 levels increased in the early stage, suggesting that the chemotherapy was ineffective. In patients who survived 12-24 months, CA 19-9 levels decreased 2 months after the treatment but increased again almost 4 months after the treatment. In the long-term survival group ( $>24$ months), most patients had a decrease in CA 19-9 levels from baseline, and no patients had RR-C $>1$ at 6 months. Therefore, patients with a rapid increase in CA 19-9 levels following an initial decrease after the initiation of chemotherapy had a poor prognosis. Long-term control of CA 19-9 levels predicts long-term prognosis. Patients with CA19-9 reduction $>44 \%$ for $>3$ months during the first 6 months had a positive association with long-term survival and distant metastases.

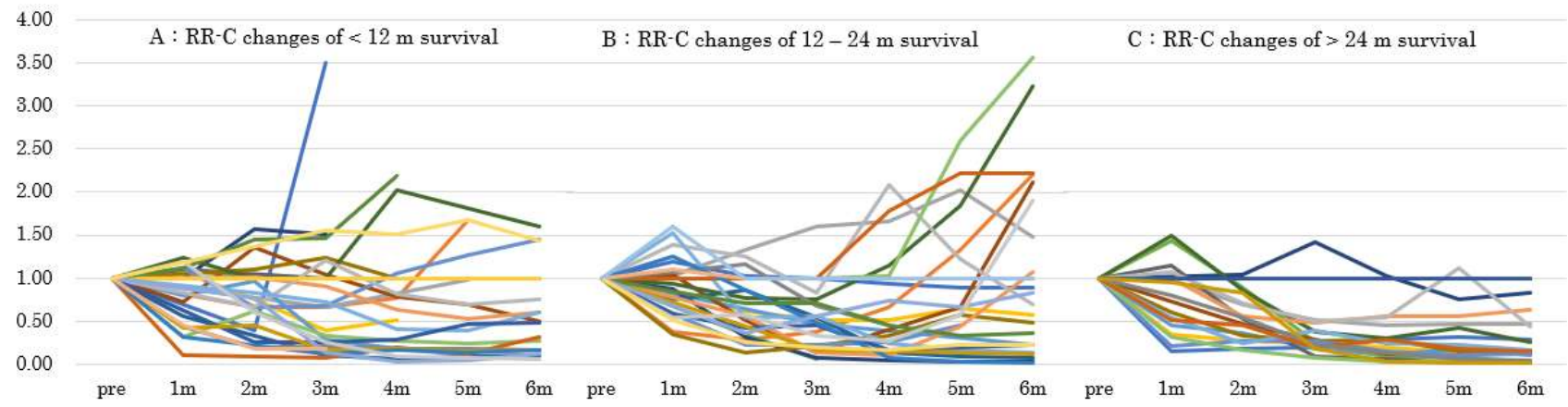

Figure 5. Analysis of the reduction ratio of CA19-9 (RR-C) in each survival. (Patients who survive within 12 months (A), from 12 months to 24 months (B), and more than 24 months (C).)

Although surgery is the only curative treatment for pancreatic cancer, invasion of the main vessels by the tumor precludes surgery. Furthermore, patients at low risk for distant metastases can receive local treatments. CRTx is associated with better long-term survival compared to CTx for LAPC patients, despite an increase in the treatment-related toxicities [28]. Distant metastases were reported after the first progression in $50-90 \%$ of patients who received CRTx $[29,30]$. Therefore, patients who have better long-term survival and low risk for distant metastases should be selected for CRTx. If the CA19-9 levels are reduced by $>44 \%$ for $>3$ months, distant metastases are unlikely, and long-term survival ( $>24$ months) can be expected. Multidisciplinary local treatments are expected to benefit these patients.

There were some limitations to our study. First, it was a single-center retrospective study, without a control group. Second, the sample size was small. Third, the CTx and CRTx regimens were not uniform. Finally, 43 follow-up CA 19-9 values (9\%) were missing.

\section{Conclusions}

In conclusion, a duration of reduced CA19-9 levels $>3$ months, rather than the rate of reduction of CA19-9 levels, during the 6 months after treatment is significantly associated with a good prognosis and predicts long-term survival. We suggest that more aggressive treatment, such as surgery and radiotherapy, will be beneficial for these patients. 
Author Contributions: Conceptualization, K.T., S.I. and H.I.; data curation, M.I., H.O., D.K., M.U., T.F. (Taito Fukuma), S.T., W.Y., Y.T., A.S. and K.I.; formal analysis, H.S. and T.F. (Taito Fukuma); investigation, K.T., S.I. and T.F. (Toshio Fujisawa); methodology, K.T., S.I., T.F. (Toshio Fujisawa) and H.I.; project administration, K.T and H.I.; software, K.T.; supervision, A.N. and H.I.; validation, S.I. and T.F. (Toshio Fujisawa); visualization, K.T. and H.I., writing-original draft, K.T.; writing-review and editing, S.I., T.F. (Toshio Fujisawa), A.N. and H.I. All authors have read and agreed to the published version of the manuscript.

Funding: This research received no external funding.

Institutional Review Board Statement: The study was conducted according to the guidelines of the Declaration of Helsinki and approved by the Institutional Review Board of Juntendo University Hospital (IRB No: 20-007).

Informed Consent Statement: Informed consent was obtained from all subjects involved in the study.

Data Availability Statement: Please refer to suggested Data Availability Statements at https:/ /www. mdpi.com/journal/cancers/instructions\#suppmaterials (accessed on 23 July 2021).

Conflicts of Interest: The authors declare no conflict of interest.

\section{References}

1. Siegel, R.L.; Miller, K.D.; Jemal, A. Cancer statistics, 2020. CA Cancer J. Clin. 2020, 70, 7-30. [CrossRef] [PubMed]

2. Wolfgang, C.L.; Herman, J.M.; Laheru, D.A.; Klein, A.P.; Erdek, M.A.; Fishman, E.K.; Hruban, R.H. Recent progress in pancreatic cancer. CA Cancer J. Clin. 2013, 63, 318-348. [CrossRef] [PubMed]

3. Yoo, C.; Shin, S.H.; Kim, K.P.; Jeong, J.H.; Chang, H.M.; Kang, J.H.; Lee, S.S.; Park, D.H.; Song, T.J.; Seo, D.W.; et al. Clinical Outcomes of Conversion Surgery after Neoadjuvant Chemotherapy in Patients with Borderline Resectable and Locally Advanced Unresectable Pancreatic Cancer: A Single-Center, Retrospective Analysis. Cancers 2019, 11, 278. [CrossRef]

4. Jang, J.Y.; Han, Y.; Lee, H.; Kim, S.W.; Kwon, W.; Lee, K.H.; Oh, D.Y.; Chie, E.K.; Lee, J.M.; Heo, J.S.; et al. Oncological Benefits of Neoadjuvant Chemoradiation With Gemcitabine Versus Upfront Surgery in Patients With Borderline Resectable Pancreatic Cancer: A Prospective, Randomized, Open-label, Multicenter Phase 2/3 Trial. Ann. Surg. 2018, 268, 215-222. [CrossRef] [PubMed]

5. Hackert, T.; Ulrich, A.; Büchler, M.W. Borderline resectable pancreatic cancer. Cancer Lett. 2016, 375, 231-237. [CrossRef]

6. Satoi, S.; Yamaue, H.; Kato, K.; Takahashi, S.; Hirono, S.; Takeda, S.; Eguchi, H.; Sho, M.; Wada, K.; Shinchi, H.; et al. Role of adjuvant surgery for patients with initially unresectable pancreatic cancer with a long-term favorable response to non-surgical anti-cancer treatments: Results of a project study for pancreatic surgery by the Japanese Society of Hepato-Biliary-Pancreatic Surgery. J. Hepato-Biliary-Pancreat. Sci. 2013, 20, 590-600. [CrossRef]

7. Rose, J.B.; Rocha, F.G.; Alseidi, A.; Biehl, T.; Moonka, R.; Ryan, J.A.; Lin, B.; Picozzi, V.; Helton, S. Extended neoadjuvant chemotherapy for borderline resectable pancreatic cancer demonstrates promising postoperative outcomes and survival. Ann. Surg. Oncol. 2014, 21, 1530-1537. [CrossRef]

8. Wolfe, A.R.; Prabhakar, D.; Yildiz, V.O.; Cloyd, J.M.; Dillhoff, M.; Abushahin, L.; Alexandra Diaz, D.; Miller, E.D.; Chen, W.; Frankel, W.L.; et al. Neoadjuvant-modified FOLFIRINOX vs. nab-paclitaxel plus gemcitabine for borderline resectable or locally advanced pancreatic cancer patients who achieved surgical resection. Cancer Med. 2020, 9, 4711-4723. [CrossRef]

9. Tsujimoto, A.; Sudo, K.; Nakamura, K.; Kita, E.; Hara, R.; Takayama, W.; Ishii, H.; Yamaguchi, T. Gemcitabine plus nab-paclitaxel for locally advanced or borderline resectable pancreatic cancer. Sci. Rep. 2019, 9, 16187. [CrossRef]

10. Lee, W.; Park, Y.; Kwon, J.W.; Jun, E.; Song, K.B.; Lee, J.H.; Hwang, D.W.; Yoo, C.; Kim, K.P.; Jeong, J.H.; et al. Reduced and Normalized Carbohydrate Antigen 19-9 Concentrations after Neoadjuvant Chemotherapy Have Comparable Prognostic Performance in Patients with Borderline Resectable and Locally Advanced Pancreatic Cancer. J. Clin. Med. 2020, 9, 1477. [CrossRef] [PubMed]

11. Boeck, S.; Stieber, P.; Holdenrieder, S.; Wilkowski, R.; Heinemann, V. Prognostic and therapeutic significance of carbohydrate antigen 19-9 as tumor marker in patients with pancreatic cancer. Oncology 2006, 70, 255-264. [CrossRef]

12. Boone, B.A.; Steve, J.; Zenati, M.S.; Hogg, M.E.; Singhi, A.D.; Bartlett, D.L.; Zureikat, A.H.; Bahary, N.; Zeh, H.J., 3rd. Serum CA 19-9 response to neoadjuvant therapy is associated with outcome in pancreatic adenocarcinoma. Ann. Surg. Oncol. 2014, 21, 4351-4358. [CrossRef] [PubMed]

13. Tzeng, C.W.; Balachandran, A.; Ahmad, M.; Lee, J.E.; Krishnan, S.; Wang, H.; Crane, C.H.; Wolff, R.A.; Varadhachary, G.R.; Pisters, P.W.; et al. Serum carbohydrate antigen 19-9 represents a marker of response to neoadjuvant therapy in patients with borderline resectable pancreatic cancer. HPB 2014, 16, 430-438. [CrossRef]

14. Groot, V.P.; Gemenetzis, G.; Blair, A.B.; Rivero-Soto, R.J.; Yu, J.; Javed, A.A.; Burkhart, R.A.; Rinkes, I.; Molenaar, I.Q.; Cameron, J.L.; et al. Defining and Predicting Early Recurrence in 957 Patients With Resected Pancreatic Ductal Adenocarcinoma. Ann. Surg. 2019, 269, 1154-1162. [CrossRef] 
15. Klaiber, U.; Schnaidt, E.S.; Hinz, U.; Gaida, M.M.; Heger, U.; Hank, T.; Strobel, O.; Neoptolemos, J.P.; Mihaljevic, A.L.; Büchler, M.W.; et al. Prognostic Factors of Survival After Neoadjuvant Treatment and Resection for Initially Unresectable Pancreatic Cancer. Ann. Surg. 2021, 273, 154-162. [CrossRef]

16. Chung, K.H.; Ryu, J.K.; Lee, B.S.; Jang, D.K.; Lee, S.H.; Kim, Y.T. Early decrement of serum carbohydrate antigen $19-9$ predicts favorable outcome in advanced pancreatic cancer. J. Gastroenterol. Hepatol. 2016, 31, 506-512. [CrossRef]

17. Nakai, Y.; Kawabe, T.; Isayama, H.; Sasaki, T.; Yagioka, H.; Yashima, Y.; Kogure, H.; Arizumi, T.; Togawa, O.; Ito, Y.; et al. CA 19-9 response as an early indicator of the effectiveness of gemcitabine in patients with advanced pancreatic cancer. Oncology 2008, 75, 120-126. [CrossRef]

18. Hess, V.; Glimelius, B.; Grawe, P.; Dietrich, D.; Bodoky, G.; Ruhstaller, T.; Bajetta, E.; Saletti, P.; Figer, A.; Scheithauer, W.; et al. CA 19-9 tumour-marker response to chemotherapy in patients with advanced pancreatic cancer enrolled in a randomised controlled trial. Lancet. Oncol. 2008, 9, 132-138. [CrossRef]

19. Reni, M.; Peretti, U.; Zanon, S.; Macchini, M.; Balzano, G.; Mazza, E.; Tamburrino, D.; Orsi, G.; Arcidiacono, P.G.; Falconi, M.; et al. Time to CA19-9 nadir: A clue for defining optimal treatment duration in patients with resectable pancreatic ductal adenocarcinoma. Cancer Chemother. Pharmacol. 2020, 85, 641-650. [CrossRef]

20. Tempero, M.A.; Uchida, E.; Takasaki, H.; Burnett, D.A.; Steplewski, Z.; Pour, P.M. Relationship of carbohydrate antigen 19-9 and Lewis antigens in pancreatic cancer. Cancer Res. 1987, 47, 5501-5503.

21. Tempero, M.A.; Malafa, M.P.; Al-Hawary, M.; Asbun, H.; Bain, A.; Behrman, S.W.; Benson, A.B., 3rd; Binder, E.; Cardin, D.B.; Cha, C.; et al. Pancreatic Adenocarcinoma, Version 2.2017, NCCN Clinical Practice Guidelines in Oncology. J. Natl. Compr. Canc. Netw. 2017, 15, 1028-1061. [CrossRef] [PubMed]

22. Eisenhauer, E.A.; Therasse, P.; Bogaerts, J.; Schwartz, L.H.; Sargent, D.; Ford, R.; Dancey, J.; Arbuck, S.; Gwyther, S.; Mooney, M.; et al. New response evaluation criteria in solid tumours: Revised RECIST guideline (version 1.1). Eur. J. Cancer 2009, 45, 228-247. [CrossRef] [PubMed]

23. Aldakkak, M.; Christians, K.K.; Krepline, A.N.; George, B.; Ritch, P.S.; Erickson, B.A.; Johnston, F.M.; Evans, D.B.; Tsai, S. Pretreatment carbohydrate antigen 19-9 does not predict the response to neoadjuvant therapy in patients with localized pancreatic cancer. HPB 2015, 17, 942-952. [CrossRef]

24. Truty, M.J.; Kendrick, M.L.; Nagorney, D.M.; Smoot, R.L.; Cleary, S.P.; Graham, R.P.; Goenka, A.H.; Hallemeier, C.L.; Haddock, M.G.; Harmsen, W.S.; et al. Factors Predicting Response, Perioperative Outcomes, and Survival Following Total Neoadjuvant Therapy for Borderline/Locally Advanced Pancreatic Cancer. Ann. Surg. 2021, 273, 341-349. [CrossRef]

25. Rose, J.B.; Edwards, A.M.; Rocha, F.G.; Clark, C.; Alseidi, A.A.; Biehl, T.R.; Lin, B.S.; Picozzi, V.J.; Helton, W.S. Sustained Carbohydrate Antigen 19-9 Response to Neoadjuvant Chemotherapy in Borderline Resectable Pancreatic Cancer Predicts Progression and Survival. Oncologist 2020, 25, 859-866. [CrossRef]

26. Lopez, N.E.; Prendergast, C.; Lowy, A.M. Borderline resectable pancreatic cancer: Definitions and management. World J. Gastroenterol. 2014, 20, 10740-10751. [CrossRef] [PubMed]

27. Hamada, C.; Okusaka, T.; Ikari, T.; Isayama, H.; Furuse, J.; Ishii, H.; Nakai, Y.; Imai, S.; Okamura, S. Efficacy and safety of gemcitabine plus S-1 in pancreatic cancer: A pooled analysis of individual patient data. Br. J. Cancer 2017, 116, 1544-1550. [CrossRef]

28. Chen, Y.; Sun, X.J.; Jiang, T.H.; Mao, A.W. Combined radiochemotherapy in patients with locally advanced pancreatic cancer: A meta-analysis. World J. Gastroenterol. 2013, 19, 7461-7471. [CrossRef]

29. Sudo, K.; Yamaguchi, T.; Ishihara, T.; Nakamura, K.; Hara, T.; Denda, T.; Tawada, K.; Imagumbai, T.; Araki, H.; Sakai, M.; et al. Phase II study of oral S-1 and concurrent radiotherapy in patients with unresectable locally advanced pancreatic cancer. Int. J. Radiat. Oncol. Biol. Phys. 2011, 80, 119-125. [CrossRef]

30. Okusaka, T.; Ito, Y.; Ueno, H.; Ikeda, M.; Takezako, Y.; Morizane, C.; Kagami, Y.; Ikeda, H. Phase II study of radiotherapy combined with gemcitabine for locally advanced pancreatic cancer. Br. J. Cancer 2004, 91, 673-677. [CrossRef] [PubMed] 